\title{
Partially-averaged Navier Stokes Model for Turbulence: Implementation and Validation
}

\author{
Sharath S. Girimaji* \\ Texas AEM University, College Station, Texas, 77843-3141, USA \\ Khaled S. Abdol-Hamid † \\ NASA Langley Research Center, Hampton, VA 23666, USA
}

\begin{abstract}
Partially-averaged Navier Stokes (PANS) is a suite of turbulence closure models of various modeled-to-resolved scale ratios ranging from Reynolds-averaged Navier Stokes (RANS) to Navier-Stokes (direct numerical simulations). The objective of PANS, like hybrid models, is to resolve large scale structures at reasonable computational expense. The modeled-to-resolved scale ratio or the level of physical resolution in PANS is quantified by two parameters: the unresolved-to-total ratios of kinetic energy $\left(f_{k}\right)$ and dissipation $\left(f_{\varepsilon}\right)$. The unresolved-scale stress is modeled with the Boussinesq approximation and modeled transport equations are solved for the unresolved kinetic energy and dissipation. In this paper, we first present a brief discussion of the PANS philosophy followed by a description of the implementation procedure and finally perform preliminary evaluation in benchmark problems.
\end{abstract}

\section{Introduction}

The optimal computation strategy for a turbulent flow dependents on the level of complexity of the flow and the degree of accuracy needed for the specific application. In a variety of production-dominated rectilinear flows (zero-pressure gradient boundary layers, straight channel flows, etc.), the current corps of Reynolds-averaged Navier-Stokes (RANS) models perform well enough to predict the mean flow statistics to within engineering accuracy. While the RANS method is computationally inexpensive, the range of flow physics it can adequately represent is severely limited due to the fact that it is a one-point closure. Many engineering applications (e.g., aeroacoustics, aeroelasticity, flow control) involve flows with large-scale unsteadiness in which coherent structures play a crucial role in determining the turbulence physics. Such flows are characterized by large correlation distances and strong non-local effects. One-point closures, by their very nature, are generally ineffective for flows with significant non-local (in space and time) effects.

Large eddy simulations (LES) typically resolve all the dynamically important scales of motion and a significant portion of the inertial scales. Thus, LES is well capable of handling wider range of turbulence physics including flows with large coherent structures. However, the increased range of flow physics and highfidelity flow details come at the expense of much greater computational effort. For engineering purposes, LES is too high a price to pay when the resolution of only the largest scales is needed.

\footnotetext{
*Associate Professor, AIAA Member.

${ }^{\dagger}$ Aerospace Engineer, AIAA Associate Fellow.

Copyright (C) 2005 by the American Institute of Aeronautics and Astronautics, Inc. The U.S. Government has a royalty-free license to exercise all rights under the copyright claimed herein for Governmental purposes. All other rights are reserved by the copyright owner.
} 
There is a clear and imminent need for turbulence computational methods of intermediate cost and degree of accuracy - relative to RANS and LES. The desire is to extract important large-scale unsteady features at minimal computational expense. Many such methods are currently under consideration: e.g., detached-eddy simulations ${ }^{1}$ (DES); hybrid RANS/LES; limited numerical scales $\operatorname{method}^{2}$ (LNS); unsteady Reynolds-averaged Navier Stokes method ${ }^{3,4}$ (URANS); and very large-eddy simulations ${ }^{5}$ (VLES).

A recent addition to this list of models of intermediate cost/accuracy is the partially-averaged NavierStokes (PANS) method of Girimaji. ${ }^{6}$ PANS is a suite of turbulence closure models of various modeled-toresolved scale ratios ranging from RANS to Navier-Stokes (direct numerical simulations). The modeledto-resolved scale ratio or the level of physical resolution in PANS is quantified by two parameters: the unresolved-to-total ratios of kinetic energy $\left(f_{k}\right)$ and dissipation $\left(f_{\varepsilon}\right)$. The unresolved stress is modeled with the Boussinessq approximation and modeled transport equations are solved for the unresolved kinetic energy and dissipation. The unresolved kinetic energy and dissipation equations for various modeled-to-resolved ratios are systematically derived from a parent RANS model. PANS models of different $f_{k}$ values require different numerical resolutions. Lower the $f_{k}$ value, the smaller is the modeled-to-resolved ratio, the greater computational effort and the more accurate the computed physics.

In this paper we will first present the PANS rationale and model equations (Section 2). In Section 3, various PANS model features are discussed and important implementation details are given with clear guidelines for the lowest possible $f_{k}$ values (leading to maximum flow resolution) that a given grid can support. Then in Section 4 we present some preliminary results and comparison against popular methods such DES and LES.

\section{The PANS method}

As seen in the introduction, in the computation of a complex turbulent flow, it is most cost-efficient to resolve only those features, including coherent structures, that are not amenable to single-point closure and represent the rest of the flow with easy-to-compute closure models. The optimum resolved-to-modeled ratio will vary from case to case depending on the flow geometry and physical effects. Therefore, it is essential to develop closure models that are valid for any arbitrary resolved-to-modeled ratio. Such is the objective of the suite of models we present in this paper. This family of models is parameterized by the modeled-toresolved kinetic energy ratio. As this model family is based on the RANS paradigm and is valid for any partial average, it is given the name Partially-averaged Navier-Stokes (PANS). We will now briefly describe the PANS closure model development. The details can be found in Girimaji. ${ }^{6}$

Incompressible Navier-Stokes equations for the instantaneous velocity $(\mathbf{V})$ and pressure $(p)$ fields are

$$
\frac{\partial V_{i}}{\partial t}+V_{j} \frac{\partial V_{i}}{\partial x_{j}}=-\frac{\partial p}{\partial x_{i}}+\nu \frac{\partial^{2} V_{i}}{\partial x_{j} \partial x_{j}} ; \quad \frac{\partial^{2} p}{\partial x_{i} \partial x_{i}}=-\frac{\partial V_{i}}{\partial x_{j}} \frac{\partial V_{j}}{\partial x_{i}} .
$$

Consider partially-averaged flow variables $-U_{i}=\left\langle V_{i}>; \quad p_{U}=\langle p\rangle-\right.$ where $\langle\cdots>$ denotes an arbitrary (implicit or explicit) filter which is constant preserving and commutes with spatial and temporal differentiation. The PANS equations are written in terms of partially averaged or filtered velocity (U) and pressure $\left(p_{U}\right)$ fields. The evolution equations are $^{7}$

$$
\begin{aligned}
\frac{\partial U_{i}}{\partial t}+U_{j} \frac{\partial U_{i}}{\partial x_{j}}+\frac{\partial \tau\left(V_{i}, V_{j}\right)}{\partial x_{j}} & =-\frac{\partial p_{U}}{\partial x_{i}}+\nu \frac{\partial^{2} U_{i}}{\partial x_{j} \partial x_{j}} \\
-\frac{\partial^{2} p_{U}}{\partial x_{i} \partial x_{i}} & =\frac{\partial U_{i}}{\partial x_{j}} \frac{\partial U_{j}}{\partial x_{i}}+\frac{\partial^{2} \tau\left(V_{i}, V_{j}\right)}{\partial x_{j} \partial x_{j}} .
\end{aligned}
$$

In the above equation, $\tau\left(V_{i}, V_{j}\right)$ is the generalized central second moment:

$$
\tau(A, B)=<A B>-<A><B>
$$


The sub-filter kinetic energy and dissipation can be defined as

$$
K_{u}=\frac{1}{2} \tau\left(V_{i}, V_{i}\right) ; \quad \varepsilon_{u}=\nu \tau\left(\frac{\partial V_{i}}{\partial x_{j}}, \frac{\partial V_{i}}{\partial x_{j}}\right) .
$$

Throughout, subscript $u$ indicate PANS statistics

When the averaging is over all scales of motion (denoted by over bar), the filtered velocity becomes the mean velocity and the SFS stress reduces to Reynolds stress $-R\left(V_{i}, V_{j}\right)$ : where $R(A, B)=\overline{A B}-\bar{A} \bar{B}$. The RANS statistics are related to their PANS counterpart according to Germano: ${ }^{7}$

$$
\bar{V}_{j}=\overline{<V_{j}>}=\overline{U_{j}} ; \quad R\left(V_{i}, V_{j}\right) \equiv \overline{V_{i} V_{j}}-\bar{V}_{i} \bar{V}_{j}=\overline{\tau\left(V_{i}, V_{j}\right)}+R\left(U_{i}, U_{j}\right)
$$

The RANS kinetic energy and dissipation are denoted by $K$ and $\varepsilon$. Averaging over all scales also leads equations (2) and $\tau\left(V_{i}, V_{j}\right)$ equations to collapse to the RANS equations. The form of the statistical hierarchy of equations is invariant of the filter, when the filtered equations are expressed in terms of the generalized central moments. ${ }^{7-9}$ This feature of the Navier-Stokes equation is given the name averaging-invariance property.

The averaging-invariance property clearly indicates that the sub-filter scale (SFS) stress $\tau\left(V_{i}, V_{j}\right)$ has the same characteristics as the Reynolds stress and similar closure strategies can be employed. As the PANS bridging model is purported for use at all degrees of physical resolution (modeled-to-resolved ratios), there must be at least as much physics incumbent in the PANS models as in some of the more advanced two-equation RANS models. Most of the popular LES closures that are algebraic in nature (zero-equation models) are too elementary to be useful in the PANS method. Years of modelling experience is incumbent in the RANS models and they have been tested widely in important engineering flows. Important issues such as tensor invariance, realizability and effects of extra strain rate (due to rotation, buoyancy, etc.) are best addressed at the level of RANS closure using well-tested techniques. ${ }^{10}$ Therefore, we base the PANS models on the RANS paradigm. As a first step, the PANS model developed in Girimaji ${ }^{6}$ is based on the Boussinesq approximation for the turbulence constitutive relation and evolution equations are solved for the unresolved kinetic energy $\left(k_{u}\right)$ and dissipation $\left(\varepsilon_{u}\right)$.

The two-equation PANS model is derived in Girimaji ${ }^{6}$ by adhering to the following guidelines: (i) The form of the bridging model closures must be invariant of the modeled-to-resolved field ratio. Averaging invariance can be a useful constraint even when the filters are slowly-varying functions of space. (ii) The physical resolution or extent of averaging of a bridging model must be appropriately quantified. For example, in URANS, the extent of averaging (implied physical resolution) is not clear. (iii) That the PANS model can achieve the required modeled-to-resolved scale ratio must be verifiable. (iv) The model must change smoothly and seamlessly from RANS to DNS as the modeled-to-resolved ratio is varied. The URANS approach not been able to demonstrate a smooth variation. ${ }^{11}$

\section{A. Closure Modeling.}

The PANS model development involves several important steps: (a) Quantification of modeled-to-resolved ratio - identification of physical-resolution parameter; (b) Specification of the physical resolution parameter. (c) Closure of PANS SFS stress, $\tau\left(V_{i}, V_{j}\right)$; (d) Modelling of the PANS transport equations; and (e) A priori verification of model behavior.

(a) In LES, the resolution is quantified in terms of the cut-off wavenumber $-\lambda_{c}$ or filter width. Larger the cut-off wavenumber, the more accurate the calculation as more scales are resolved exactly. The cut-off wavenumber enters the calculations via filtering and subgrid-stress constitutive relationship and thus controls the physical resolution. For PANS it is important to identify the corresponding physical resolution parameter that (i) quantifies the filtering, and (ii) appears in the closure model to ensure that the required resolution is achieved. In Girimaji ${ }^{6}$ it is shown that the extent of PANS averaging - relative to RANS - can be best quantified using the unresolved-to-total ratios of kinetic energy $\left(f_{k}\right)$ and dissipation $\left(f_{\varepsilon}\right)$ :

$$
f_{k}=\frac{K_{u}}{K}, \quad f_{\varepsilon}=\frac{\varepsilon_{u}}{\varepsilon} .
$$

3 of 14

American Institute of Aeronautics and Astronautics Paper 2005-0502 
These two parameters are the PANS modeled-to-resolved ratio quantification or resolution-control parameters. Note that turbulence physics - large scales contain most of the kinetic energy and much of the dissipation occurs in the smallest scales - dictates $0 \leq f_{k} \leq f_{\varepsilon} \leq 1$. Smaller the $f_{k}$, greater is the physical resolution: $f_{k}=1$ represents RANS and $f_{k}=0$ indicates DNS. Unity value of $f_{\epsilon}$ implies that the RANS and PANS unresolved small scales are identical.

(b) In order to perform a PANS computation, the resolution control parameters must be specified. It is generally desirable to have the physical resolution vary as a function of space as in DES. Rapid spatialvariation of physical resolution will be considered in future works as it introduces an additional complication of a filtering operation that does not commute with spatial differentiation. In this paper, we will assume that the specified spatio-temporal variation of the implied PANS filter is slow compared to that of flow variables. The prescription of $f_{k}$ and $f_{\varepsilon}$ is discussed in the next section.

(c) As mentioned earlier, in this work we will focus on a two-equation PANS closure. When the Boussinesq approximation is invoked in conjunction with averaging-invariance requirement for arbitrary filters, we get:

$$
\tau\left(V_{i}, V_{j}\right)=-\nu_{u} S_{i j} ; \text { where } \nu_{u}=C_{\mu} \frac{K_{u}^{2}}{\varepsilon_{u}} .
$$

Fixed-point analysis (e.g. Girimaji ${ }^{10}$ ) has clearly shown that the asymptotic energetics of unresolved scales does not depend on the value of $C_{\mu}$. Hence, for the sake of simplicity, we maintain the PANS $C_{\mu}$ at the RANS value.

(d) The derivation of the model evolution equations for $K_{u}$ and $\varepsilon_{u}$ as functions of $f_{K}$ and $f_{\varepsilon}$ is described in detail in Girimaji. ${ }^{6}$ The two-equation PANS model can be summarized as

$$
\begin{aligned}
\frac{d K_{u}}{d t} & =P_{u}-\varepsilon_{u}+\frac{\partial}{\partial x_{j}}\left(\left(\frac{\nu_{u}}{\sigma_{k u}}+\nu\right) \frac{\partial K_{u}}{\partial x_{j}}\right) \\
\frac{d \varepsilon_{u}}{d t} & =f_{k}\left(C_{e 1} \frac{P_{u} \varepsilon_{u}}{K_{u}}-C_{e 2}^{*} \frac{\varepsilon_{u}^{2}}{K_{u}}\right)+\frac{\partial}{\partial x_{j}}\left(\left(\frac{\nu_{u}}{\sigma_{e u}}+\nu\right) \frac{\partial \varepsilon_{u}}{\partial x_{j}}\right) .
\end{aligned}
$$

The modified model coefficients are

$$
C_{e 2}^{*} \equiv C_{e 1}+\frac{f_{k}}{f_{\varepsilon}}\left(C_{e 2}-C_{e 1}\right) ; \quad \sigma_{k, \epsilon u} \equiv \sigma_{k, \epsilon} \frac{f_{k}^{2}}{f_{\epsilon}} .
$$

The coefficients $C_{e 1}, C_{e 2}, \sigma$ 's are those of the parent RANS model, which in this case is the standard twoequation closure. The values for the various model constants used in our works are: $C_{e 1}=1.44 ; C_{e 2}=$ $1.92 ; \sigma_{k}=1.0 ; \sigma_{\varepsilon}=1.3$.

Overall, the form of the equations are clearly invariant to averaging. The resolution-control parameter manifests only via the modified model coefficients.

(e) Whether the PANS model can achieve the required level of modeled-to-resolved ratio depends on the production-to-dissipation ratio $-P_{u} / \varepsilon_{u} \cdot{ }^{12}$ The larger the value of $P_{u} / \varepsilon_{u}$, the more energetic are the unresolved scales. In a bridging model, the energy content of the unresolved fluctuations should decrease as the resolution goes from RANS $\left(f_{k}=1\right)$ to DNS $\left(f_{k}=0\right)$. When the implied cut-off is in the dissipation range, $P_{u} / \varepsilon_{u}$ should go to unity as all energy cascaded into the dissipation scales is immediately expended without any accumulation. The long-time global behavior $P_{u} / \varepsilon_{u}$ as a function of resolution can be best evaluated from a fixed point analysis of equations (8) in the absence of transport (which does not create of destroy energy). A detailed analysis is given in Girimaji et al ${ }^{12}$ and only the important results are presented here. At the weak-equilibrium turbulence fixed point, we have only one non-trivial fixed point:

$$
\left(\frac{P_{u}}{\varepsilon_{u}}\right)_{\lim t \rightarrow \infty}=\frac{C_{e 2}^{*}-1}{C_{e 1}-1}=1+\left(\frac{f_{k}}{f_{\varepsilon}}\right) \frac{C_{e 2}-C_{e 1}}{C_{e 1}-1} .
$$

Asymptotically, $P_{u} / \varepsilon_{u}$ varies linearly with $f_{k}$ - going from the RANS value to DNS value of unity. The PANS SFS velocity field progressively gets weaker with smaller $f_{k}$ as required. On the contrary in URANS, 
wherein the model coefficients are constants at RANS values, the only non-trivial fixed point is the same as in RANS. Hence, the URANS unresolved scales will be as energetic as the RANS scales irrespective of implied resolution as was found by Khorrami et al. ${ }^{3}$ It is shown in Girimaji et al ${ }^{12}$ that the trivial fixed point of RANS leads to $K=\varepsilon=0$. This corresponds to DNS. Thus, URANS can asymptotically go only to RANS or DNS as was found by Hedges et al. ${ }^{11}$

\section{PANS features and implementation}

The PANS method represents a new modelling paradigm that is distinct from LES, DES, URANS and hybrid methods. Now, we will identify some of the PANS distinguishing features.

1) The PANS decomposition of the velocity field is based on kinetic energy content rather than on cut-off wavenumber. The filter demarcating calculated (resolved) and modeled (unresolved) motion is implied rather than explicit. PANS can be viewed as LES with an implicit filter and a two-equation sub-filter closure.

2) The parameter $f_{k}$ determines the unresolved-to-total kinetic energy and thereby sets the cut-off between resolved and unresolved scales. Thus, the resolution of the flow is controlled by suitably specifying $f_{k}$.

3) The parameter $f_{\varepsilon}$ dictates the extent of overlap between the energy-containing and dissipation ranges in the given simulation. The Reynolds number of the simulation is controlled by suitably specifying $f_{\varepsilon}$. If there is a clear separation between energy-containing and dissipation scales (high Reynolds number flow), then $f_{\varepsilon}=1$. If there is no significant separation between the two ranges (low Reynolds number flow), then $f_{\varepsilon} \approx f_{k}$. For intermediate Reynolds numbers, the $f_{\varepsilon}$ value will be between the two limits. In LES closures, it is difficult to control the Reynolds number of the flow.

4) The parameters $f_{k}$ and $f_{\varepsilon}$ can be constant throughout the computational domain (some kind of fractional RANS) or vary as a function of space and time (in the spirit of DES).

5) The SFS (sub-filter scale) model is independent of grid spacing, and dependent only on the parameters $f_{k}$ and $f_{\varepsilon}$. Thus, the physical resolution is decoupled from numerical resolution. This results in a model of higher fidelity than zero-equation algebraic closures based on grid spacing.

6) The cut-off corresponding to given values of $f_{k}$ and $f_{\varepsilon}$ cannot be easily estimated a priori. Thus, the numerical grid required for given $f_{k}$ and $f_{\varepsilon}$ can only be found using a grid independence study. This can be the same type of grid-refinement studies used to determine the appropriate grid for a RANS simulation.

7) Due to the nature of PANS no explicit filtering operation is necessary. The SFS closure model is designed to ensure the right balance between the resolved and modeled kinetic energies.

\section{A. PANS implementation}

Any computational tool that is capable of simulating different modeled-to-resolved ratios must be made up of two important components: high-fidelity physical model; and, effective and efficient numerical implementation scheme. The first component is a closure model possessing the turbulence physics to ensure that the right amount of energy is modeled. The PANS method described above is theoretically proven to yield the desired level of unresolved kinetic energy. The second component ensures that the full benefits of the physical model are realized, resulting in the best possible physical accuracy at a given level of computational effort. We will now briefly describe a scheme to effectively implement PANS. More details on the scheme and a sample implementation can be found in. ${ }^{13}$

The objective of the implementation scheme is to determine the lowest $f_{k}$ and $f_{\varepsilon}$ a given grid can support for a specified flow geometry and boundary conditions. Recall that, the lower the values of the PANS parameters, the more accurate the computations as more scales are resolved. In our analysis, we will restrict our consideration to high Reynolds number turbulence with the implicit cut-off located anywhere except in the dissipation range. This should cover almost all cases of practical interest. Since the dissipation scales are not resolved in these cases, it can be assumed that $f_{\varepsilon} \approx 1$.

The physical criterion used in assessing the lowest $f_{k}$ that can be supported at a given grid location is

5 of 14

American Institute of Aeronautics and Astronautics Paper 2005-0502 
derived from the requirement that the grid-size should be larger than the smallest resolved length scale. But, what is the smallest resolved length scale for a given $f_{k}$ ? This can be estimated by adapting Kolmogorov type arguments to resolved motion. The smallest resolved length scale $\left(\eta_{r}\right)$ is likely to be determined by local dissipation and local eddy viscosity:

$$
\eta_{r} \sim\left(\frac{\nu_{u}^{3}}{\varepsilon}\right)^{\frac{1}{4}}
$$

This is the resolved-field equivalent of the Kolmogorov scale. From equation (7) we can write (noting $f_{\varepsilon}=1$, $\left.\varepsilon_{u}=\varepsilon\right)$

$$
\nu_{u}=C_{\mu} \frac{K_{u}^{2}}{\varepsilon_{u}} \approx C_{\mu} f_{k}^{2} \frac{K^{2}}{\varepsilon} .
$$

The resolved-field Kolmogorov scale is

$$
\eta_{r}=C_{\mu}^{0.75} f_{k}^{1.5} \Lambda
$$

where $\Lambda=K^{1.5} / \varepsilon$ is the Taylor scale of turbulence. The smallest resolved length scale in PANS is related to $f_{k}$ and the Taylor scale of turbulence which is to be expected.

A typical guideline in DNS is that the grid-spacing should be of the order of the Kolmogorov length scale. A similar requirement for PANS would lead to

$$
\eta_{r}=C_{\mu}^{0.75} f_{k}^{1.5} \Lambda \sim \Delta
$$

where $\Delta$ is the smallest grid dimension. Thus, the smallest $f_{k}$ a grid can support at a given location is

$$
f_{k}(\mathbf{x})=\frac{1}{\sqrt{C_{\mu}}}\left(\frac{\Delta}{\Lambda}\right)^{\frac{2}{3}} \approx 3\left(\frac{\Delta}{\Lambda}\right)^{\frac{2}{3}}
$$

In order to judiciously specify $f_{k}(\mathbf{x})$ we must know the spatial distribution of the Taylor microscale or, equivalently, the distributions of the total kinetic energy and dissipation. Since, this is not known a priori we propose two possible approaches. First is an in situ approach, in which we start the computation with a plausible $f_{k}$ distribution and compute the total energy and dissipation by suitable averaging. Then, the $f_{k}(\mathbf{x})$ value is continually updated using equation (15). This method will be similar to dynamic Smagorinsky approach which requires on-the-fly averaging to determine the Smagorinsky closure constant. While this approach may appear to be physically correct, we expect it to be susceptible to statistical errors in the averaging process, especially if there are no homogeneous directions. At this stage of PANS development, we propose a second approach which is simpler and more robust even if not as theoretically correct as the first method. We suggest a one-time RANS calculation for specifying the $f_{k}(\mathbf{x})$ distribution which will then be maintained constant in time. While a RANS calculation may not yield very accurate results, it can serve as a guide for $f_{k}$ specification. The inaccuracy of RANS will not affect the accuracy ensuing PANS calculation too much. The inaccurate RANS can only cause the $f_{k}$ distribution to be less than fully optimal, resulting in the use of slightly different $f_{k}$ than the grid can optimally support. Based on the second method, we now prescribe a four-step implementation scheme:

1. For the flow of interest, the (most affordable) grid is first generated. Then a RANS calculation is performed. This can be done quite expeditiously. This is called the pre-processing computational step.

2. The RANS data is then used to determine the Taylor-scale distribution.

3. The lowest possible $f_{k}$ distribution is then found from equation (15).

4. Once the $f_{k}$-field is specified, the full calculation can the be performed by modifying the model coefficients as per PANS closure. The $f_{k}$-field is held constant throughout the computation.

This procedure is shown in a flow chart in Fig. 1. Results obtained from implementation of this scheme will be shown in the next section. 


\section{Results}

The results are presented in two parts. In the first part, we present preliminary results from fixed $f_{k}$ calculations. In the second part, the results from spatially varying (temporally constant) $f_{k}$ computation are shown.

Fixed $f_{k}$ COMPUTATIONs. Our first objective is to show that PANS models produce progressively better results with decreasing $f_{k}$. This demonstration is best achieved with constant $f_{k}$ computations. Several large-scale constant- $f_{k}$ PANS computations of flow past square cylinder, high and low-Reynolds number flow past circular cylinder, flow in a square cavity, and flow over a surface mounted cube have been completed. In all these studies, the commercial CFD code FLUENT is used with the model coefficients appropriately modified depending on the specified resolution control parameter. Standard wall-function approach is used for the first near-wall grid point.

In the square cylinder study, the PANS results of various physical resolutions are compared with URANS, LES $^{14}$ and experimental data. ${ }^{15}$ In Table 1, the Strouhal number, mean coefficient of drag, rms (root-mean square) of drag, rms of lift are given for three PANS computations of different $f_{k}$ values. The $f_{k}=1$ computation can be viewed as the URANS method. All the PANS and URANS computations are performed on the same grid to ensure that the difference in calculated results is only due to the modeling effects. Except in the case of Strouhal number, URANS compares poorly with LES or experiments. With decreasing $f_{k}$ (increasing physical resolution), the PANS results approach the experimental values for all quantities considered. The $f_{k}=0.4$ case is nearly as accurate as LES which employs twice as many grid points. The centerline mean streamwise velocity profile obtained from the three PANS calculations are compared in Fig. 2 against experimental and LES results. Clearly, with decreasing $f_{k}$, the PANS profile approaches the experimental data resulting in excellent agreement. The reason for the improved performance with decreasing $f_{k}$ can be surmised from flow structures shown in Fig. 3. More and more of the flow features are resolved with decreasing $f_{k}$. Whereas, in $f_{k}=1$ case, the flow appears nearly laminar, a variety of smallscale turbulent structures can be seen in $f_{k}=0.4$ case. Another dramatic change resulting from decreasing $f_{k}$ can be seen in Fig. 4 where instantaneous iso-surfaces of transverse (Z) velocity are shown for $f_{k}=1$ and $f_{k}=0.7$ cases. In the $f_{k}=1$ case, there is absolutely no transverse velocity indicating a two-dimensional flow. In the $f_{k}=0.7$ case, there is clearly a great deal of three-dimensional motion.

The $C_{p}$ profiles computed from various $f_{k}$ computations of flow past circular cylinder are shown in Fig. 5 for high Reynolds number flow and in Fig. 6 for low Reynolds number case. Experimental, DES and LES data are also shown for comparison. In the DES calculation, the turbulence model is activated only past the laminar separation point. Hence, the DES computation is hard-wired to predict separation correctly. In our PANS calculation, the turbulence model is always activated. The PANS model does miss the separation point, but captures the remainder of the details quite well. Even here, it is quite evident that the results improve substantially with decreasing $f_{k}$.

The conclusion from the constant $f_{k}$ studies is clearly that the PANS can go from RANS to experiments smoothly as $f_{k}$ value is decreased - thus achieving our objective.

Spatially-VARYing $f_{k}$ COMPUtATion. Having established that PANS model suite does perform as required with decreasing constant- $f_{k}$, we now compute with spatially varying $f_{k}$. The objective, as in DES, is to use RANS model (no fluctuating scales resolved) in the near-wall regions and progressively resolve more scales of motion in the areas of interest. For these computations we use the NASA Langley code PAB3D. The test case is an axisymmetric Mach 0.55 jet with a nozzle exit diameter $\left(D_{j}\right)$ of $2 \mathrm{in}$ (experiment of Bridges and Brown $\left.^{16}\right)$.The freestream Mach number is 0.01 . The computational mesh is a three-dimensional grid with 120 cells in the circumferential direction. The domain is divided into 48 parts. Grids are clustered around the solid surfaces and the shear layer (Fig. 7). Uniform grid is used in the region $2<x / D_{j}<12$. The value of $y^{+}$at the first grid point is maintained between 0.2 and 2.0. PANS computation is performed at three different grid resolutions: coarse (500,000 cells); medium $(2,000,000$ cells $)$ and fine $(4,000,000$ cells $)$. The 
$f_{k}$-distribution is specified using the procedure outlined in the previous section. Unsteady RANS calculation is also performed for comparison. Figure 8 shows an instantaneous two-dimensional snap shot from the medium resolution PANS computation. The jet breakdown as it interacts with the external flow is clearly seen. In Fig. 9, the time-averaged stream-wise mean velocity profiles obtained from RANS and PANS are compared against experimental data. Clearly, the PANS result is much closer to the experimental data than the RANS calculation. It should be mentioned that the instantaneous and time-averaged RANS results were very similar. This shows that the RANS model does not capture important unsteady effects. The RANS core, due to insufficient mixing, extends farther downstream than observed in experimental data. In Fig. 10, the centerline mean stream-wise velocity obtained from PANS simulations of different resolutions are compared against experimental data. The coarse simulation results overpredict the jet penetration, consistent with poor turbulent mixing characteristics. The medium and fine resolution simulations, on the other hand, agree well with the experiment. The fact that the medium and fine results are in close agreement indicates the grid insensitivity of the results. Finally, in Fig. 11, we compare the total centerline kinetic energy with the unresolved kinetic energy as computed in the fine-resolution PANS. The total experimental centerline kinetic energy is also shown. The two important observations are: (i) the total PANS kinetic energy agrees reasonably well with the experimental data; and (ii) most of the kinetic energy is in the resolved scales.

In this paper, we provide a brief introduction to the theory and practice of the PANS method of turbulence simulation. The initial results are encouraging. This material is based upon work supported by NASA under NN104AA41G.

\section{References}

${ }^{1}$ Spalart, P. R., Trends in turbulence treatments, AIAA 2000-2306, Fluids 2000, Denver, CO, 2000.

${ }^{2}$ Batten, P., Goldberg, U., and Chakravarthy, S., LNS - An approach towards embedded LES, AIAA $2002-0427$.

${ }^{3}$ Khorrami, M. R., Singer, B., Berkman, M. E., "Time-accurate simulations and and acoustic analysis of slat free shear layer", AIAA Journal, Vol. 40, 2002, pp.1284-1291.

${ }^{4}$ Khorrami, M. R., Singer, B., and Lockard, D., Time-accurate simulations and and acoustic analysis of slat free shear layer: Part 2, AIAA 2002-2579, AIAA/CEAS Aeroacoustics Conference and Exhibit, Breckenridge, CO, 2002.

${ }^{5}$ Speziale, C. G., Computing Non-Equilibrium Flows with Time-Dependent RANS and VLES, 15th ICNMFD, 1996.

${ }^{6}$ Girimaji, S. S., Partiall-averaged Navier-Stokes (PANS) method for turbulence: A RANS to DNS bridging method, Submitted to Journal of Applied Mechanics.

${ }^{7}$ Germano, M., Turbulence: the filtering approach, Journal of Fluid Mechanics, Vol. 238, 1992, pp.325-336.

${ }^{8}$ Lilly, D. K., On the application of the eddy-viscosity concept in the inertial sub-range of turbulence, NCAR, Boulder. Manuscript 123, 1966.

${ }^{9}$ Deardorff, J.W., A numerical study of three-dimensional channel flow at large Reynolds numbers, Journal of Fluid Mechanics, Vol. 41, 1970, pp. 453-480.

${ }^{10}$ Girimaji, S. S., "Pressure-strain correlation modeling of complex turbulent flows". Journal of Fluid Mechanics, Vol. 422, 2000, pp. 91-123.

${ }^{11}$ Hedges, L. S., Travin, A. K., and Spalart, P. S., Detached-eddy simulation over a simplified landing gear, Journal Fluids Engineering, Vol.124, 2002, pp.413-423.

${ }^{12}$ Girimaji, S. S., Sreenivasan, R. and Jeong, E., PANS turbulence model for seamless transition between RANS and LES: Fixed point analysis and preliminary results, FEDSM 2003-45336, Proceedings of FEDSM'03, 2003 4-th ASME-JSME Joint Fluids Engineering Conferences, July 13 - 16, Honolulu, HA, USA.

${ }^{13}$ Abdol-Hamid, K. S. and Girimaji, S. S., A two-stage procedure toward efficient implementation of PANS and other hybrid turbulence models, NASA-TM 2004-21360.

${ }^{14}$ Sohankar, A. and Davidson, L., "Large Eddy Simulations of flow past a square cylinder: Comparison of different sub-grid models". ASME Journal of Fluid Engineering, Vol. 122, 2000, p.39.

${ }^{15}$ Lyn, D. A., Einav, S., Rodi, W. and Park, J. H., "A Laser-Doppler Velocimetry study of the ensemble-averaged characteristics of the turbulent wake of a square cylinder", Journal of Fluid Mechanics, Vol. 304, 1992, p.285.

${ }^{16}$ Bridges J., and Brown, C., Parametric testing on single flow hot jet, AIAA 2004-2824. 
Table 1. Square Cylinder Flow Predictions

\begin{tabular}{cccccc} 
Case & $R e / 10^{3}$ & $S_{t}$ & $\left(C_{D}\right)_{\text {mean }}$ & $\left(C_{D}\right)_{r m s}$ & $\left(C_{L}\right)_{r m s}$ \\
\hline PANS $\left(f_{k}=1.0\right)$ & 22 & 0.132 & 1.44 & 0.0022 & 0.27 \\
PANS $\left(f_{k}=0.7\right)$ & 22 & 0.133 & 1.77 & 0.129 & 0.994 \\
PANS $\left(f_{k}=0.4\right)$ & 22 & 0.130 & 1.97 & 0.216 & 1.19 \\
LES & 22 & $0.126-0.132$ & $2.03-2.32$ & $0.16-0.20$ & $1.23-1.54$ \\
Exp Lyn & 21.4 & 0.132 & 2.1 & - & - \\
Exp Durao & 14 & 0.138 & - & - & - \\
Exp McLean & $15-40$ & - & - & - & 1.4 \\
Exp Berman & $5.8-32$ & 0.130 & - & - & 1.2 \\
Exp Norberg & 13 & 0.132 & 2.16 & - & - \\
Exp Luo & 34 & 0.13 & 2.2 & 0.18 & 1.2
\end{tabular}

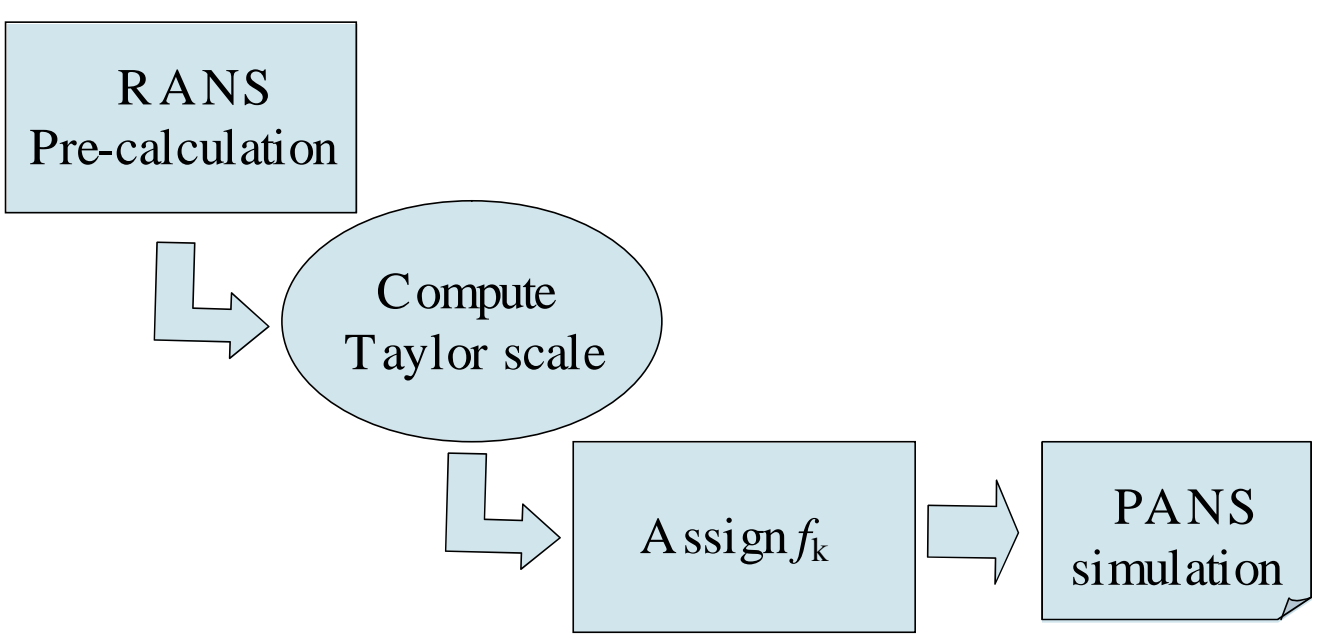

Figure 1. Four-step implementation scheme. 


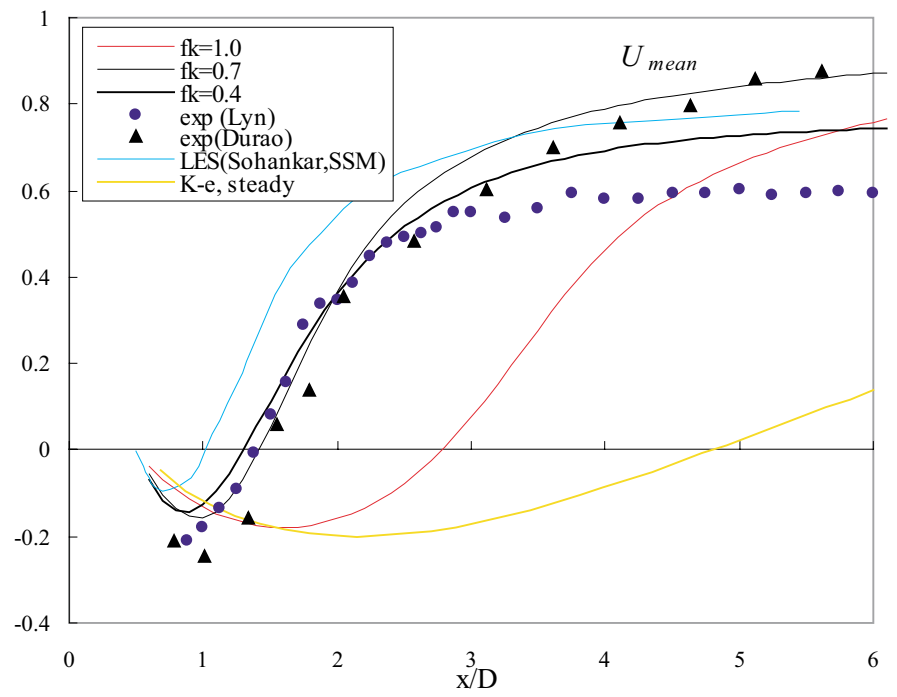

Figure 2. Streamwise mean velocity profiles along the centerline from various experiments and simulations.

$f_{\mathrm{k}}=1.0$

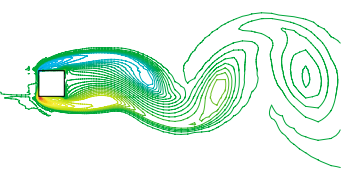

$20 \cdot 16 \cdot 12-8 \cdot-4 \cdot 1.6-0.800 .81 .648 \quad 8121620$ $f_{\mathrm{k}}=0.7$

$f_{\mathrm{k}}=0.4$

Figure 3. Instantaneous contour plots of Z-vorticity .

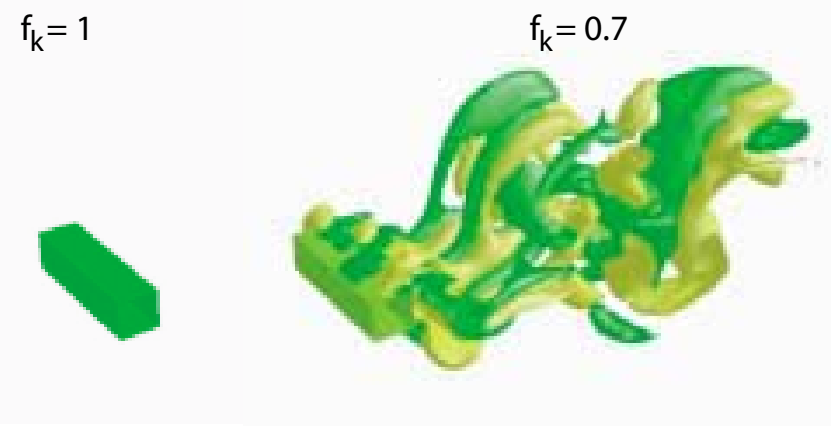

Figure 4. Instantaneous iso-surface of Z-velocity.

10 of 14 


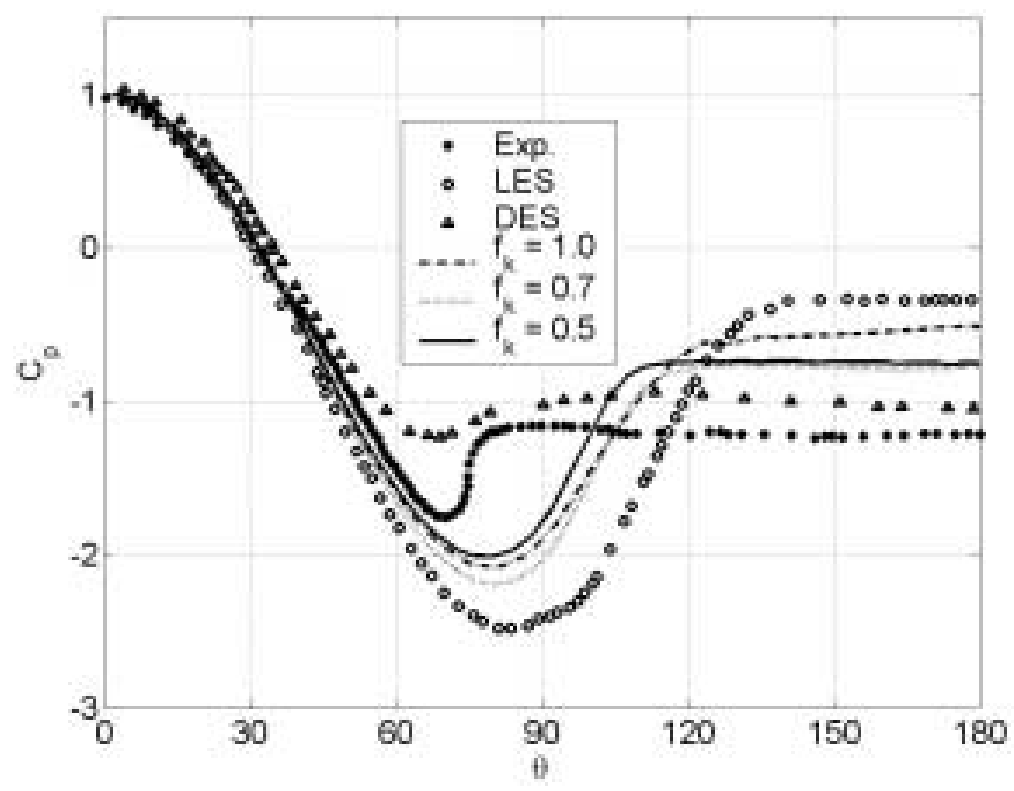

Figure 5. Profiles of Coefficient of Pressure Distribution Along the Cylinder Surface for Various $f_{k}$ Values at $\operatorname{Re}_{D}=1.4 \times 10^{5}$. , Experimental Data from Achenbach for Flow Reynolds Number of $\operatorname{Re}_{D}=1 \times 10^{5} ; *$, LES Data from Wang et al. at $R e_{D}=10^{6}$;, , DES Data from Travin et al. (run LS8); -, Data from PANS with $f_{k}=0.5$; $\cdots$, Data from PANS with $f_{k}=0.7 ;-\cdot-\cdot$, Data from PANS with $f_{k}=1.0$.

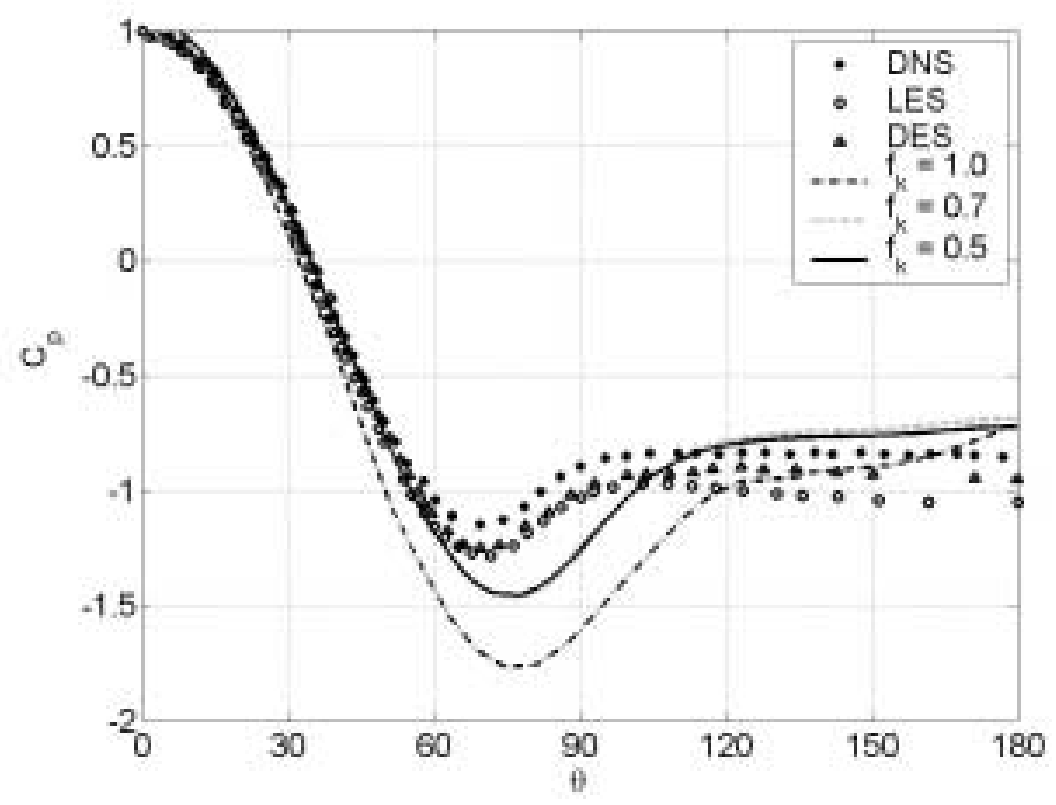

Figure 6. Profiles of Coefficient of Pressure Distribution Along the Cylinder Surface for Various $f_{k}$ Values at $R e_{D}=3900 . *$, DNS Data from Ma et al.; ○, LES Data from Breuer; $\triangle$, DES Data from Hansen and Forsythe; - , Data from PANS with $f_{k}=0.5 ; \cdots$, Data from PANS with $f_{k}=0.7 ;-\cdot-\cdot-$, Data from PANS with $f_{k}=1.0$. 


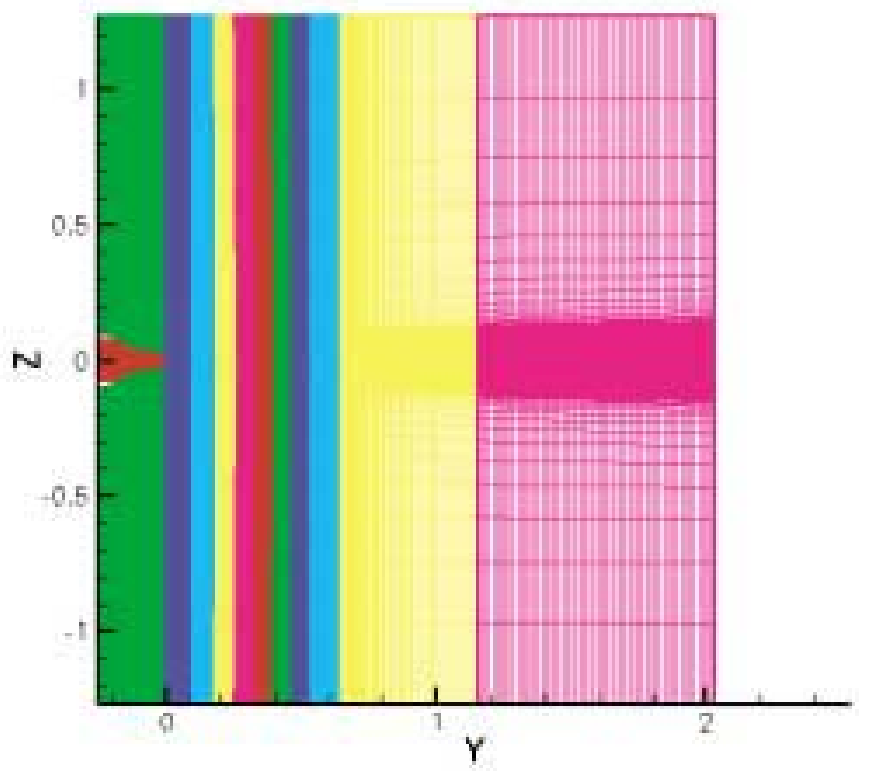

Figure 7. Computational grid for axisymmetric jet.

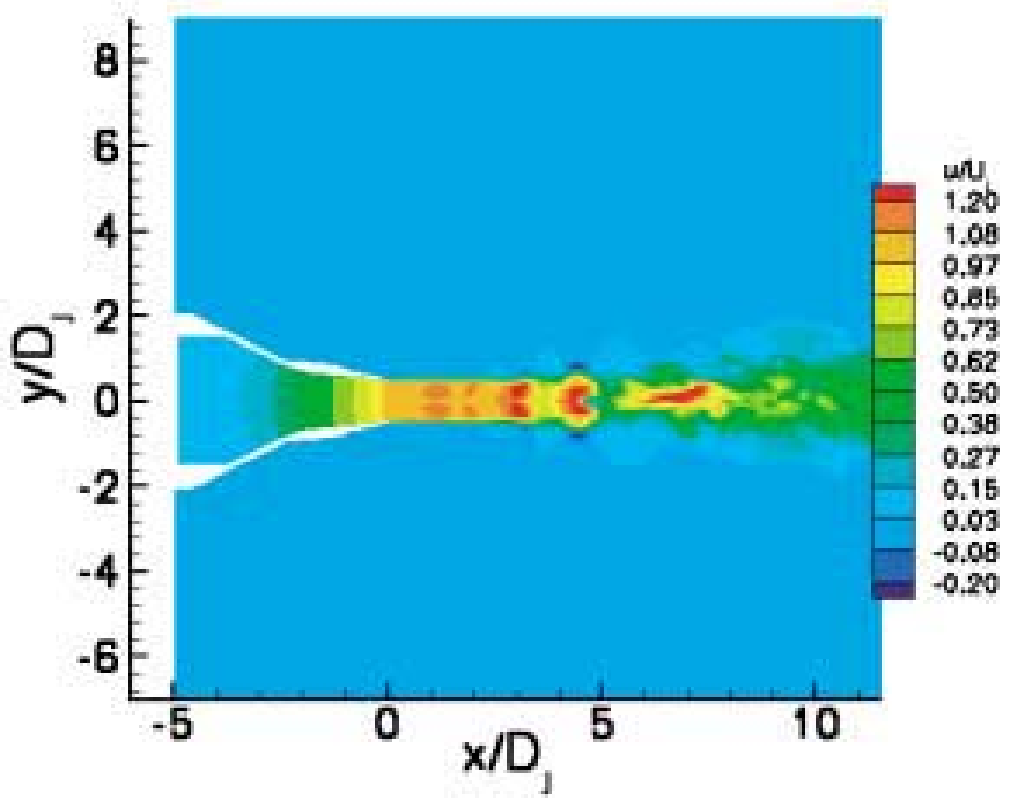

Figure 8. Instantaneous PANS velocity field.

12 of 14

American Institute of Aeronautics and Astronautics Paper 2005-0502 
DATA

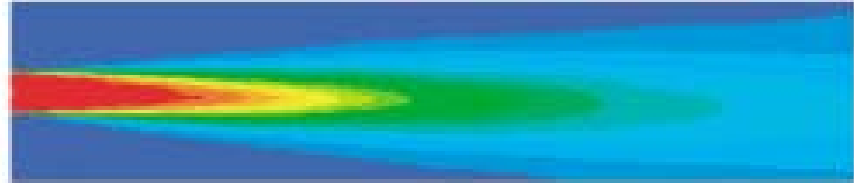

PANS

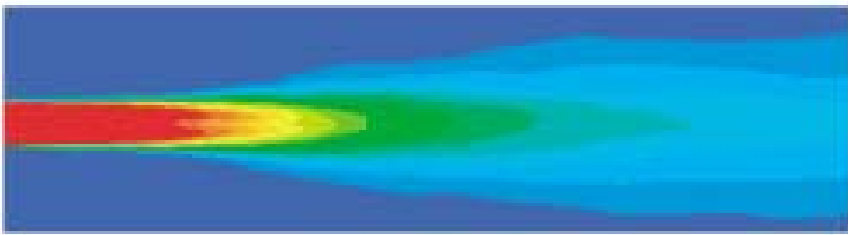

RANS

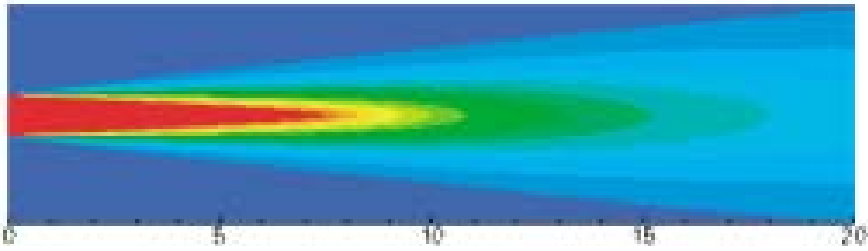

Figure 9. Axial mean velocity field.

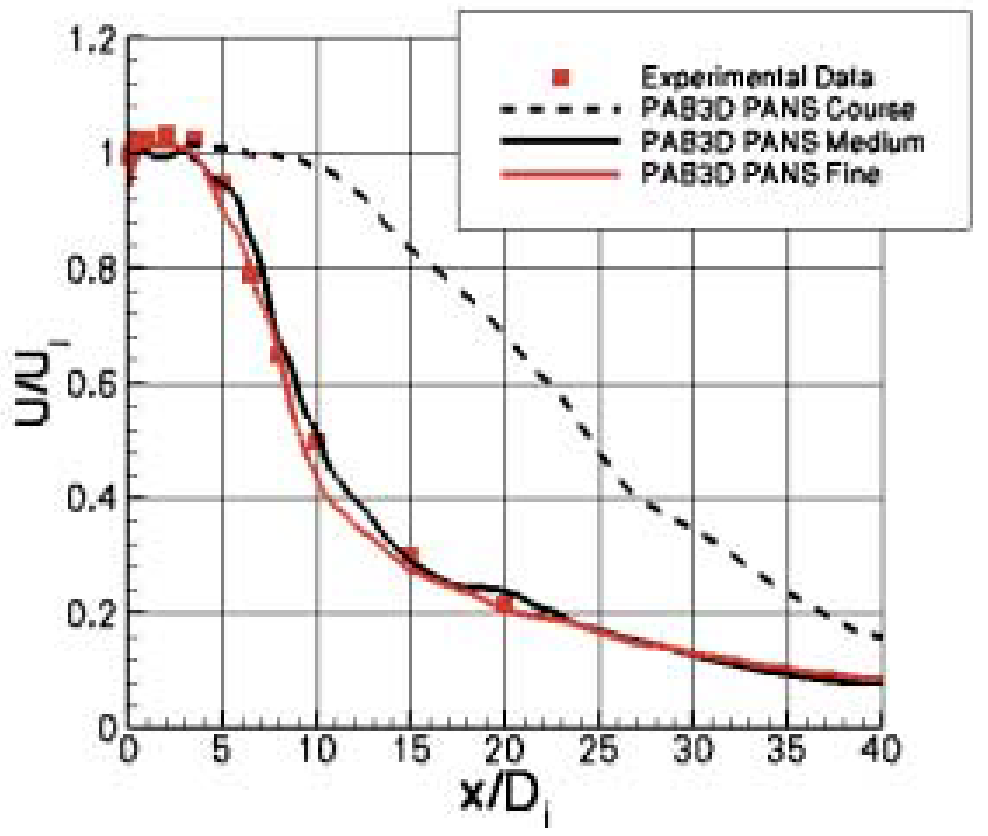

Figure 10. Centerline mean axial velocity .

13 of 14 


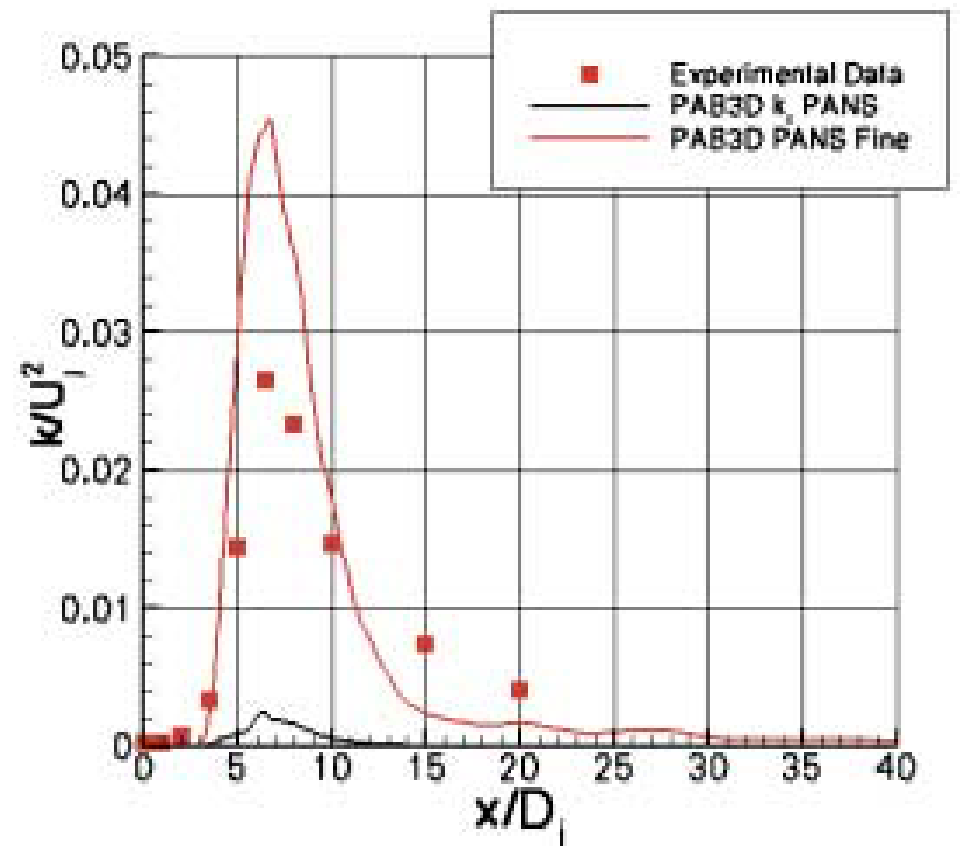

Figure 11. Centerline turbulent kinetic energy .

14 of 14 